\title{
Spin wave resonance excitation in ferromagnetic films using planar waveguide structures
}

Y. V. Khivintsev, L. Reisman, J. Lovejoy, R. Adam, C. M. Schneider, R. E. Camley, and Z. J. Celinski

Citation: Journal of Applied Physics 108, 023907 (2010);

View online: https://doi.org/10.1063/1.3435318

View Table of Contents: http://aip.scitation.org/toc/jap/108/2

Published by the American Institute of Physics

\section{Articles you may be interested in}

Ferromagnetic resonance linewidth in metallic thin films: Comparison of measurement methods Journal of Applied Physics 99, 093909 (2006); 10.1063/1.2197087

Propagating spin wave spectroscopy in a permalloy film: A quantitative analysis Applied Physics Letters 83, 972 (2003); 10.1063/1.1597745

Ferromagnetic resonance and damping properties of $\mathrm{CoFeB}$ thin films as free layers in MgO-based magnetic tunnel junctions

Journal of Applied Physics 110, 033910 (2011); 10.1063/1.3615961

Spin waves in micro-structured yttrium iron garnet nanometer-thick films Journal of Applied Physics 117, 17D128 (2015); 10.1063/1.4916027

Eddy-current effects on ferromagnetic resonance: Spin wave excitations and microwave screening effects Journal of Applied Physics 119, 163903 (2016); 10.1063/1.4948302

The design and verification of MuMax3

AIP Advances 4, 107133 (2014); 10.1063/1.4899186

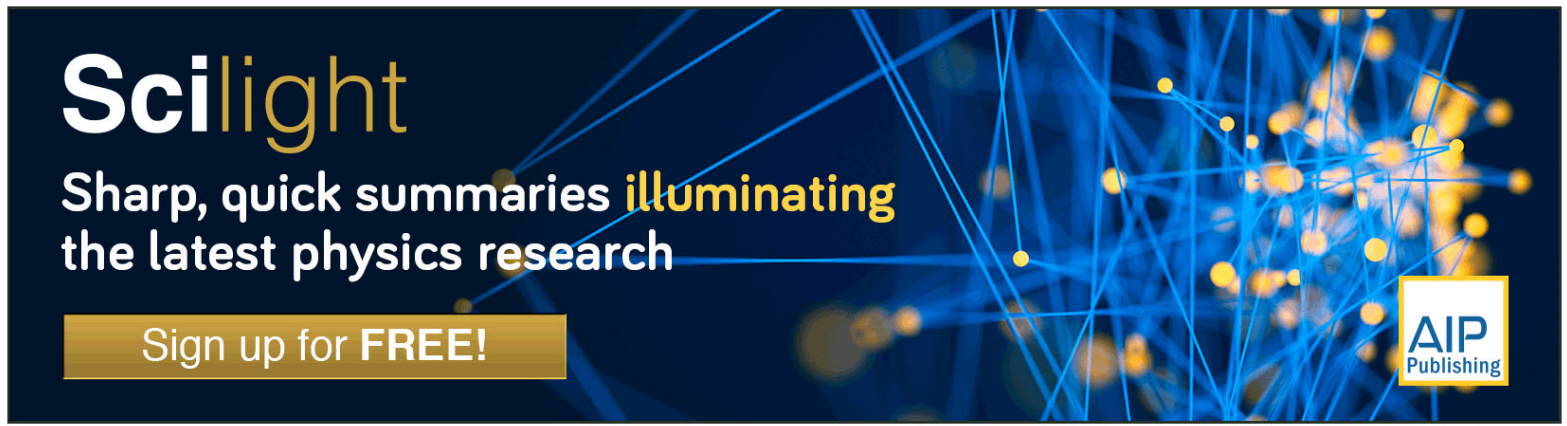




\title{
Spin wave resonance excitation in ferromagnetic films using planar waveguide structures
}

\author{
Y. V. Khivintsev, ${ }^{1,2}$ L. Reisman, ${ }^{1}$ J. Lovejoy, ${ }^{1}$ R. Adam, ${ }^{3}$ C. M. Schneider, ${ }^{3}$ R. E. Camley, ${ }^{1, a)}$ \\ and Z. J. Celinski ${ }^{1}$ \\ ${ }^{1}$ Center for Magnetism and Magnetic Nanostructures, University of Colorado at Colorado Springs, \\ Colorado Springs, Colorado 80918, USA \\ ${ }^{2}$ Kotel'nikov Institute of Radio-engineering and Electronics of Russian Academy of Sciences, Saratov \\ Branch, Saratov 410019, Russia \\ ${ }^{3}$ Institute of Solid State Research IFF-9, Research Center Jülich, Jülich D-52425, Germany
}

(Received 30 March 2010; accepted 29 April 2010; published online 21 July 2010)

\begin{abstract}
We explore a new geometry allowing effective excitation of the lowest antisymmetric standing spin wave mode in ferromagnetic metallic films with symmetrical boundary conditions. The approach is based on the use of a coplanar waveguide with the ferromagnetic film, Permalloy (Py), playing the role of the signal line. In addition, we study a signal line which is a sandwich of Py inside two nonmagnetic metallic films. We find that the thickness and conductivity of the metal films can significantly alter the amount of absorption, at ferromagnetic resonance, between the symmetric and antisymmetric spin wave modes. The experimental results are supported by numerical calculations indicating the origin of the strength of the absorption. (c) 2010 American Institute of Physics. [doi:10.1063/1.3435318]
\end{abstract}

\section{INTRODUCTION}

The method of ferromagnetic resonance (FMR) has been widely used for decades in the study of magnetic films and layered structures. ${ }^{1-6}$ It is a very powerful technique for the investigation of spin dynamics and for determining the magnetic parameters of the films and is widely used in magnetism.

In the conventional (or so-called cavity) FMR technique, the microwave magnetic driving field is close to being uniform across the thickness of the thin ferromagnetic film. This is true also in a more recent technique utilizing planar waveguide structures where the magnetic film is directly over or under the signal line of the waveguides. ${ }^{7-19}$ The uniform field produces strong coupling with the lowest spin wave resonance (SWR) mode; the coupling reduces with the order of the SWR mode. In addition, if the film has the symmetrical boundary conditions for spins on the surfaces (same conditions on both film surfaces) then the microwave field couples only with the symmetrical SWR modes and excitation of the antisymmetrical SWR modes becomes impossible. As a result in most of the FMR experiments only the lowest SWR mode is observed.

However, higher order SWR modes are also attractive for studies, especially because they provide the possibility to investigate effects of the exchange interaction in magnetic films. In particular, the SWR modes are a very convenient tool to determine the exchange stiffness. ${ }^{20}$ Moreover, the higher order SWR modes can be interesting for applications in microwave signal processing. Recently, there have been a number of reports of microwave devices utilizing the FMR corresponding to the excitation of the lowest SWR mode, see, for example, recent reviews. ${ }^{21,22}$ Taking into account

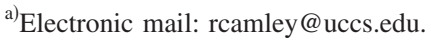

that the SWR frequencies increase with the order of the mode, the use of the higher order SWR modes in such devices can allow signal processing at higher frequencies without the need of applying large bias fields.

Previously it was suggested that an asymmetry in the magnetic boundary conditions of the magnetic film could be introduced by placing additional thin layers of different magnetic materials on its surfaces. ${ }^{23}$ This approach avoids the symmetry rule for coupling of the uniform driving field with the SWR modes and would allow observation of the higher SWR modes. However, this method requires a significantly more complicated analysis of the experimental data because it must take into account the additional magnetic layers. Also, this approach does not significantly change the coupling in favor of the higher order SWR modes and the lowest SWR mode usually remains as the most efficiently excited mode. This is undesirable for microwave applications, such as a turnable notch filter, where a single resonance response is required.

In this paper, we propose a new approach which creates a microwave driving magnetic field that is antisymmetric about the midplane of the magnetic film. This can be used for effective excitation of the antisymmetrical SWR modes in ferromagnetic films with symmetrical magnetic boundary conditions. The approach is based on the use of a coplanar waveguide with the ferromagnetic film playing the role of the signal line. We present and discuss experimental results on the coplanar waveguide structures with Permalloy (Py) films. In addition, we explore a signal line which is a sandwich of Py inside two nonmagnetic metal films. We find that the thickness and conductivity of the metal films can significantly alter the amount of absorption between symmetric and antisymmetric spin wave modes. 


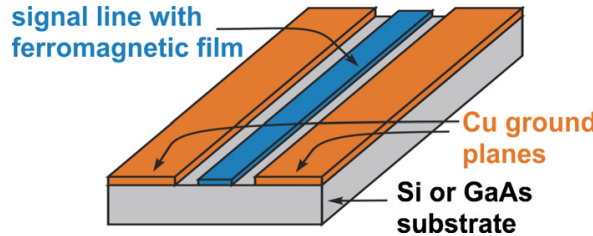

FIG. 1. (Color online) Diagram of a coplanar waveguide with an integrated ferromagnetic film in the signal line.

\section{BACKGROUND}

We consider a coplanar waveguide with the signal line composed of a ferromagnetic film and metallic films in various layering configurations-see Fig. 1. Also, we assume that the ferromagnetic film has symmetrical boundary conditions for spins on the surfaces and that its thickness is much less than the skin depth. An external static magnetic bias field is applied along the signal line. The oscillating magnetic field, $\mathrm{h}_{\mathrm{rf}}$, arising from currents in the signal line is always perpendicular to the bias field.

The power absorption in the coplanar waveguide, a measure of how efficiently a given mode is excited, is proportional to the overlap integral across the thickness of the ferromagnetic film

$$
\omega \int_{-t / 2}^{\mathrm{t} / 2} \overrightarrow{\mathrm{m}}(\mathrm{y}) \cdot \overrightarrow{\mathrm{h}}_{\mathrm{rf}}(\mathrm{y}) \mathrm{dy},
$$

where $\overrightarrow{\mathrm{m}}(\mathrm{y})$ is the distribution of the dynamic magnetization across the thickness, $\overrightarrow{\mathrm{h}}_{\mathrm{rf}}(\mathrm{y})$ is the distribution of the driving magnetic field, and $t$ is the thickness of the film measured along the $y$ axis. As will be discussed below we expect $\vec{h}_{\mathrm{rf}}(\mathrm{y})$ to be primarily in the $\mathrm{x}$ direction (horizontal). The geometry is defined in Fig. 2. The distribution $\mathrm{m}_{\mathrm{x}}(\mathrm{y})$ depends on the boundary conditions for the spins on the surfaces of the film and the order of the SWR mode. For symmetrical boundary conditions $\mathrm{m}_{\mathrm{x}}(\mathrm{y})$ is symmetrical (even SWR modes) or antisymmetrical (odd SWR modes) about the midplane of the Py film. The lowest frequency SWR mode is even and followed by an odd mode with a higher frequency, etc. Figure 2 shows $\mathrm{m}_{\mathrm{x}}(\mathrm{y})$ for the two lowest SWR modes in the case of the film with both surfaces unpinned. In the long-wavelength limit, the SWR frequencies corresponding to this situation can be calculated as ${ }^{20}$

$$
\mathrm{f}_{\mathrm{SWR}}=\gamma \sqrt{\left\{\mathrm{H}_{0}+\mathrm{D}[\pi(\mathrm{n}-1) / \mathrm{t}]^{2}\right\}\left\{\mathrm{H}_{0}+4 \pi \mathrm{M}_{\mathrm{S}}+\mathrm{D}[\pi(\mathrm{n}-1) / \mathrm{t}]^{2}\right\}},
$$

where $\gamma$ is gyromagnetic ratio, $\mathrm{H}_{0}$ is the bias magnetic field, $\mathrm{M}_{\mathrm{S}}$ is the effective saturation magnetization, $\mathrm{D}$ is the exchange stiffness, and $n=0,1,2, \ldots$.

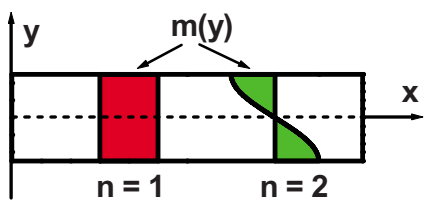

FIG. 2. (Color online) Schematic illustration of the two lowest frequency spin wave modes. The $n=1$ mode is even about the horizontal midplane of the film, while the $n=2$ mode is odd.

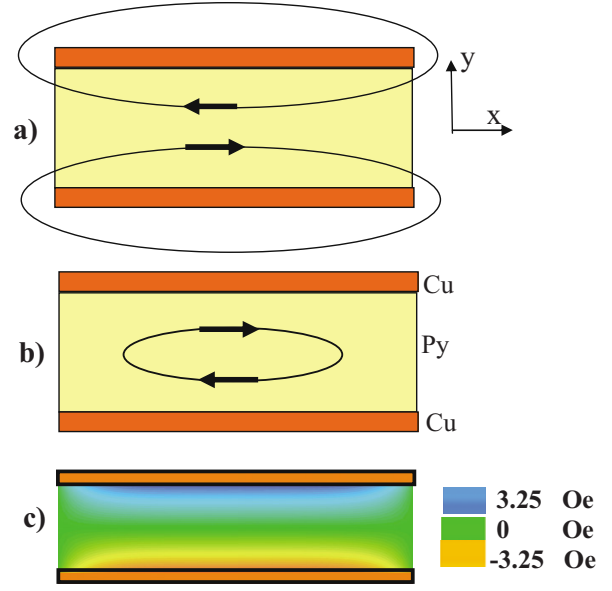

FIG. 3. (Color online) (a) Schematic illustration of $h_{r f}$ fields from currents in outer Cu films; (b) Schematic illustration of $h_{r f}$ fields from currents the Py film; and (c) realistic calculation of the $\mathrm{h}_{\mathrm{x}}(\mathrm{x}, \mathrm{y})$ for a 6/50/6 (thicknesses in nanometer) $\mathrm{Cu} / \mathrm{Py} / \mathrm{Cu}$ structure.

We can understand the general behavior of $\mathrm{h}_{\mathrm{x}}(\mathrm{y})$ through some simple arguments. In Fig. 3(a) we illustrate the fields produced by currents in the $\mathrm{Cu}$ which are flowing into the page. From Ampere's law we expect the $h_{x}$ field from the currents in either $\mathrm{Cu}$ film to be independent of $\mathrm{y}$, for $\mathrm{y}$ values significantly smaller that the width of the signal line. If the two $\mathrm{Cu}$ films carry the same current, the fields from the $\mathrm{Cu}$ films will cancel in the Py. In Fig. 3(b) we show the fields produced by the currents just in the Py film. It is easily seen that these fields will be antisymmetric around the midplane. The details of the field distribution obviously depend on the conductivity and current distribution in the various layers. In Fig. 3(c) we show an example of a realistic $h_{x}(y)$ calculated using ANSOFT HFSS $^{\mathrm{TM}}$ where the conductivity of $\mathrm{Cu}$ is 5.87 $\times 10^{7} \mathrm{~S} / \mathrm{m}$ and the conductivity of Py is $2.0 \times 10^{6} \mathrm{~S} / \mathrm{m}$ and the total power in the structure is $2 \mathrm{~W}$. The calculations were carried out with mesh sizes ranging from 3 to $10 \mathrm{~nm}$. The structure is $6 \mathrm{~nm} \mathrm{Cu} / 50 \mathrm{~nm} \mathrm{Py} / 6 \mathrm{~nm} \mathrm{Cu}$ and the width of the signal line is $50 \mu \mathrm{m}$. As expected the $\mathrm{x}$ component of the net $\mathrm{h}$ field is purely antisymmetric about the midplane.

Using the simple arguments above, we can predict some general behaviors. In the case of a ferromagnetic film placed above (or under) a thick metallic signal line the distribution $\mathrm{h}_{\mathrm{rf}}(\mathrm{y})$ is very close to uniform throughout the thickness of the ferromagnetic film-see Fig. 4(a). As a result, such a geometry allows excitation only for the even SWR modes, and the maximum absorption takes place for the lowest frequency SWR mode. In order to make excitations possible for odd SWR modes in the ferromagnetic film with symmetrical boundary conditions, the symmetry of $h_{x}(y)$ must be changed. This can be done by splitting the signal line and placing the ferromagnetic film in the middle-see Fig. 4(b). In such a case $h_{x}(y)$ becomes antisymmetric about the midplane of the film - the fields from the outer conductors cancel and one is left with only the antisymmetric field produced by the current in the ferromagnet (here, we neglect the effect of the asymmetry of the electromagnetic conditions due to the difference in the dielectric constant for the wafer and air). Thus, this geometry provides the possibility for an 


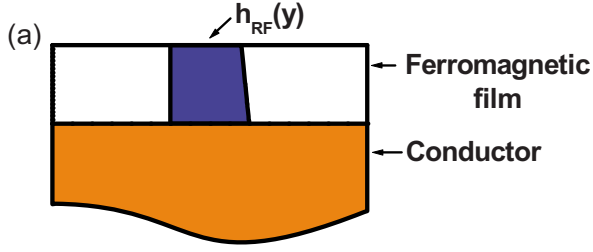

(b)

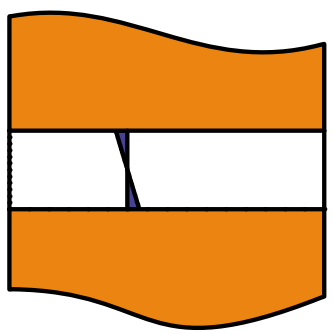

(c)

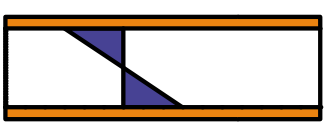

FIG. 4. (Color online) Distribution of the microwave magnetic driving field across the ferromagnetic film thickness for different cases: (a) ferromagnet on the top of the thick $\mathrm{Cu}$ signal line, (b) ferromagnet between two thick $\mathrm{Cu}$ films in the signal line, and (c) ferromagnet between two thin $\mathrm{Cu}$ films in the signal line.

effective excitation of odd SWR modes. In contrast, even SWR modes are now forbidden because $h_{r f}$ is antisymmetric and the even modes are symmetric about the midplane of the magnetic film.

In the last geometry (metal film/Py film/metal film) the thickness of nonmagnetic conductive layers can play an important role. Indeed, if the nonmagnetic conductive layers are equal in thickness to each other and much thicker or significantly more conductive than the ferromagnetic film, then most of the microwave current is carried by these conductive layers. The microwave fields produced by the conductive layers then significantly cancel each other inside the thin ferromagnetic film as depicted in Fig. 4(b). This reduces $\mathrm{h}_{\mathrm{x}}$ in the ferromagnet and thus the value for the integral in Eq. (1) becomes smaller and the absorption is reduced. In order to redistribute the microwave current between the nonmagnetic conductor and the ferromagnetic metal in favor of the latter the thickness of the nonmagnetic conductive layers must be reduced [see Fig. 4(c)]. Thus, one should expect the most efficient excitation of the odd SWR modes when the signal line will consist only of the ferromagnetic metallic layer.

\section{EXPERIMENT: SAMPLES AND MEASUREMENTS}

We fabricated a set of the coplanar waveguides with a $\mathrm{Cu} / \mathrm{Py} / \mathrm{Cu}$ sandwich structure as the signal line on $\mathrm{Si}$ or GaAs wafers using photolithography and magnetron sputtering. The $\mathrm{Cu}$ ground planes and the ends of the signal lines were either 300 or $500 \mathrm{~nm}$ thick. The signal lines were $\mathrm{Cu} /$ $\mathrm{Py} / \mathrm{Cu}$ sandwiches. Also, in some initial experiments $\mathrm{Ti} /$ $\mathrm{Py} / \mathrm{Cu}$ sandwiches were used to increase adhesion of the Py to the substrate.

Using $\mathrm{Cu}$ above and below the Py films is an attempt to create the same boundary conditions for spins on both surfaces of the Py film. The thickness of the Py film was varied

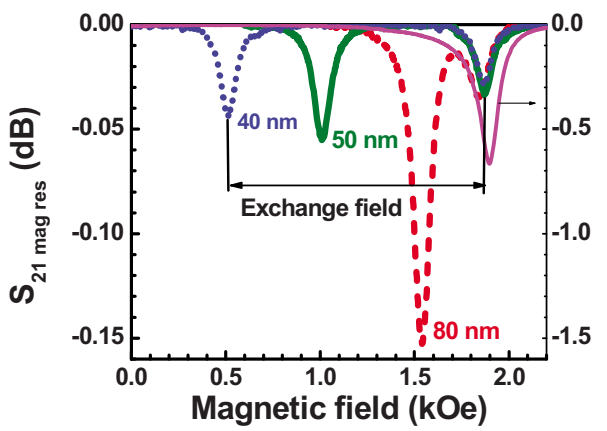

FIG. 5. (Color online) FMR responses for the samples with 40 (dotted line), 50 (solid line), and $80 \mathrm{~nm}$ (dashed line) thick Py films and $6 \mathrm{~nm}$ thick $\mathrm{Cu}$ bottom and top layers at the pumping frequency of $14 \mathrm{GHz}$. The thin solid line shows the FMR response in the case of a $\mathrm{Cu}(6 \mathrm{~nm}) / \mathrm{Py}(50 \mathrm{~nm}) / \mathrm{Cu}(6$ $\mathrm{nm}$ ) sandwich on the top of the $300 \mathrm{~nm}$ thick $\mathrm{Cu}$ signal line.

from 20 to $80 \mathrm{~nm}$. The thicknesses of the bottom and top $\mathrm{Cu}$ layers were varied from 1 to $9 \mathrm{~nm}$. The signal lines were $50 \mu \mathrm{m}$ wide with $35 \mu \mathrm{m}$ wide gaps to the ground planes. This results in a structure which has an impedance of about $50 \Omega$ on $\mathrm{Si}$ and GaAs wafers. The length of the magnetic part of the signal lines was $2.5 \mathrm{~mm}$.

Microwave measurements were carried out using a vector network analyzer along with a microwave probe station. The S-parameters were measured in the field-swept mode (the pumping frequency is kept constant and S-parameters are measured as a function of the external magnetic field) or in the frequency-swept mode (the external magnetic field is kept constant and S-parameters are measured as a function of the frequency).

In order to extract the FMR absorption, we used only the $\mathrm{S}_{21}$ parameter, neglecting the effect of the resonances on the impedance of the coplanar waveguide. In this case, the FMR response, representing the imaginary part of the microwave magnetic susceptibility, can be defined simply as a difference between magnitudes of $\mathrm{S}_{21}\left(\mathrm{~S}_{21} \mathrm{mag}\right)$ at and out of resonance. ${ }^{12}$ Thus we calculated the FMR response as

$$
S_{21 \text { mag res }}=S_{21 \text { mag }}-S_{21 \text { mag background, }}
$$

where $S_{21}$ mag background is the magnitude of the "background" $\mathrm{S}_{21}$, a result of the dielectric and conductive losses as well as the mismatching effect in the coplanar waveguide. The background value is the maximum of $S_{21}$ mag, measured away from the resonances, in the magnetic field range of $0.25-3.5$ kOe.

\section{RESULTS AND DISCUSSION}

Figure 5 shows the FMR responses obtained in the fieldswept mode at $14 \mathrm{GHz}$ for samples with various thicknesses of the Py film and the same thickness $(6 \mathrm{~nm})$ of the $\mathrm{Cu}$ bottom and top layers. One can see two distinct absorption peaks for each sample: a high field peak and a low field peak. The high field peaks for the samples with different Py thicknesses are almost identical and their positions nearly coincide with the position of the single FMR peak in the case of the Py film on top of a thick $\mathrm{Cu}$ signal line. The position of the low field peak strongly depends on the Py thickness: the thinner the Py film, the lower the resonance field for this 


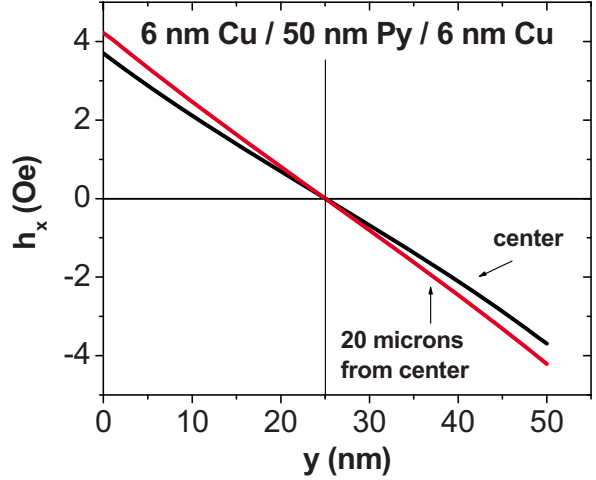

FIG. 6. (Color online) Calculated dependence of $h_{x}$ as function of the vertical position $\mathrm{y}$ in the Py for $\mathrm{x}=0$ (center of signal line) and $\mathrm{x}=20 \mu \mathrm{m}$ (near the edge of the signal line). The frequency is $12 \mathrm{GHz}$ and $\mathrm{H}_{0}=0$. The structure is $6 \mathrm{~nm} \mathrm{Cu} / 50 \mathrm{~nm} \mathrm{Py} / 6 \mathrm{~nm} \mathrm{Cu}$. The $\mathrm{h}_{\mathrm{x}}(\mathrm{y})$ signal is antisymmetric.

peak. Based on this we conclude the high field peak is effectively the "uniform mode" normally observed in FMR. The low field peak is an exchange mode where the resonant field depends on thickness according to Eq. (2). So this structure already exhibits a large absorption from the exchange mode compared to that of the uniform mode. As indicated earlier this is due to the fact that $\mathrm{h}_{\mathrm{x}}(\mathrm{y})$ is antisymmetric about the midplane at $y=25 \mathrm{~nm}$. In Fig. 6 we present realistic calculations for this field, both at $\mathrm{x}=0$, at the center of the signal line and $x=20 \mu \mathrm{m}$, near the edge of the signal line. Clearly the field distribution is antisymmetric over most of the signal line.

Figure 7 shows the FMR frequencies for both observed absorption peaks as a function of the bias magnetic field for the samples with various Py thicknesses. The $20 \mathrm{~nm}$ Py data comes from a $\mathrm{Ti} / \mathrm{Py} / \mathrm{Cu}$ sample. In this figure we also plot the results of a calculation using Eq. (2) for the lowest SWR mode $(n=1)$ with the following parameters for Py: $4 \pi \mathrm{M}_{S}$ $=10.5 \mathrm{kG}$ and $\gamma=2.9 \mathrm{GHz} / \mathrm{kOe}$. One can see good agreement between the calculations and the experimental data points for the high field absorption peaks confirming that the

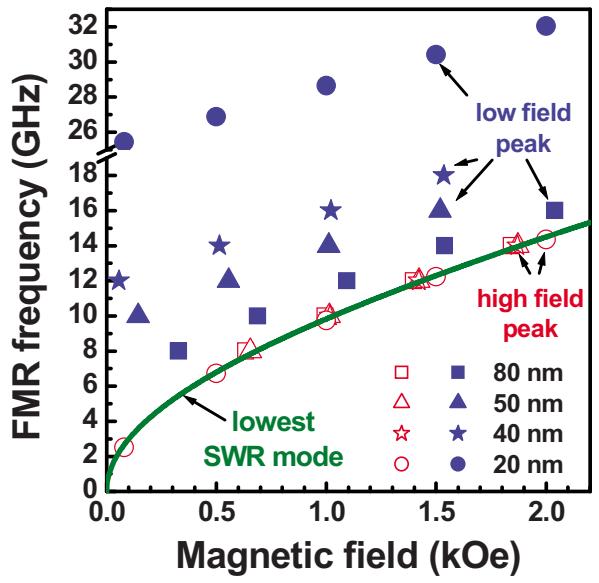

FIG. 7. (Color online) FMR frequencies vs applied magnetic field for the samples with 20 (circles), 40 (stars), 50 (triangles), and 80 (squares) thick Py layers and $6 \mathrm{~nm}$ thick $\mathrm{Cu}$ bottom and top layers (except sample with 20 nm thick Py where $2 \mathrm{~nm}$ of $\mathrm{Ti}$ and $10 \mathrm{~nm}$ of $\mathrm{Cu}$ were used as bottom and top layers, respectively). Open and filled dots correspond to the high and low field responses, respectively. Solid line indicates calculations for the lowest SWR mode in the case of unpinned surfaces.

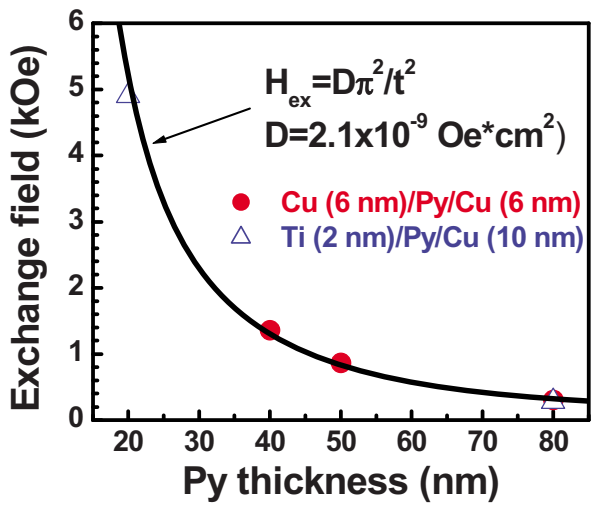

FIG. 8. (Color online) Exchange field as a function of Py thickness for the samples with $6 \mathrm{~nm} \mathrm{Cu}$ bottom and top layers (filled circles) and $2 \mathrm{~nm} \mathrm{Ti}$ under and $10 \mathrm{~nm} \mathrm{Cu}$ over layers (open triangles). Solid line indicates calculations for Py film with unpinned surfaces.

high field FMR response corresponds to the lowest frequency SWR mode, the uniform mode in the unpinned case.

The behavior of the low field FMR response follows the theoretical prediction for the next $(n=2)$ resonance, the lowest antisymmetrical SWR mode. In order to show that, we plotted the calculated and measured exchange field as a function of the Py thickness in Fig. 8. The exchange field was defined as the difference in resonance fields between the first and second SWR modes. The calculation was done using Eq. (2) with the known value of the Py exchange stiffness D $=2.1 \times 10^{-9} \mathrm{Oe} \mathrm{cm}^{2}{ }^{24,25}$ In the experiment the measured exchange field differs slightly (not more than 5\%) for the various pumping frequencies, so the figure represents the averaged values. One can see good agreement between the experiment and the theory.

In summary, when the signal line consists of a symmetric $\mathrm{Cu} / \mathrm{Py} / \mathrm{Cu}$ structure we observe the two lowest SWR modes such as: the lowest symmetrical $(\mathrm{n}=1)$ and the lowest antisymmetrical $(n=2)$ modes. The comparison with the theory shows that the behavior of the Py film is consistent with unpinned spins on the surfaces.

In the symmetric case, the efficiency of excitation, represented by the magnitude of the peaks, was higher for the antisymmetrical mode than for the symmetrical mode (see Fig. 5). However, as discussed above the latter should not be observed at all. One of the possible explanations for the excitation of the symmetrical SWR mode in our experiment is that an asymmetry is introduced by the electromagnetic boundary conditions. On the bottom the signal line is bounded by the dielectric substrate, while the top surface is bounded by air. The effect of the asymmetry may lead to an asymmetry in the distribution of the microwave current across the signal line; the microwave current could be higher at the $\mathrm{Cu} /$ dielectric interface. One could try to compensate for such an asymmetry and eliminate the coupling to the symmetrical SWR mode by adjusting the thickness of the metallic bottom and top layers or by using materials with different conductivities. Another possible explanation is that the spin pinning conditions are different at the two boundaries. This could be true because the $\mathrm{Cu} / \mathrm{Py}$ interface is likely to be different from the $\mathrm{Py} / \mathrm{Cu}$ interface, due to growth order and microscopic growth processes, for example. 


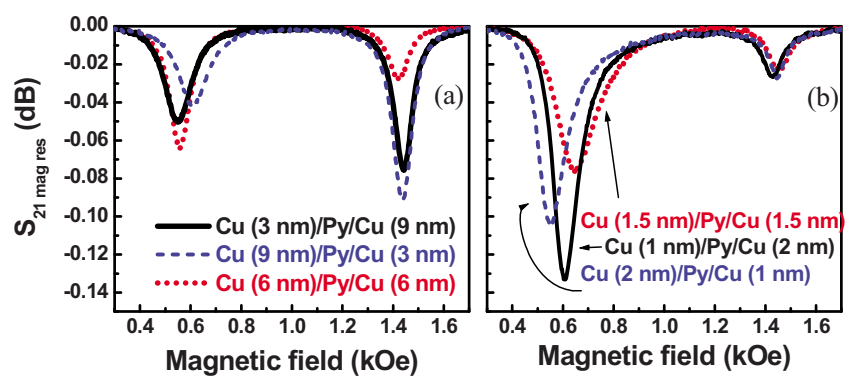

FIG. 9. (Color online) FMR response of the samples with a $50 \mathrm{~nm}$ thick Py layer and different thicknesses of the $\mathrm{Cu}$ under and over layers: (a) $12 \mathrm{~nm}$ of $\mathrm{Cu}$ in total and (b) $3 \mathrm{~nm}$ of $\mathrm{Cu}$ in total. Pumping frequency is $12 \mathrm{GHz}$.

In order to examine the consequences of changing the thicknesses of the metallic $\mathrm{Cu}$ layers we performed experiments on structures with various thicknesses of bottom and top layers. Figure 9 shows the experimental data for two classes of structures. In all cases the thickness of Py in the signal line was $50 \mathrm{~nm}$. One set of structures has $\mathrm{Cu}$ cladding layers with a total thickness of $12 \mathrm{~nm}$ [Fig. 9(a)], the second set has a total thickness for the $\mathrm{Cu}$ cladding of $3 \mathrm{~nm}$ [Fig. 9(b)]. Taking into account that the conductivity of bulk Py is about 30 times smaller than that of bulk $\mathrm{Cu}$ the first case represents the situation when most of the microwave current is concentrated in the $\mathrm{Cu}$, whereas, the second case corresponds to a situation when the current is distributed more uniformly between the $\mathrm{Cu}$ and Py layers.

The structures with thick and thin $\mathrm{Cu}$ claddings demonstrate qualitatively different behavior of the FMR peaks as the asymmetry in the thickness of the $\mathrm{Cu}$ layers is introduced. For the thick $\mathrm{Cu}$ [Fig. 9(a)] the antisymmetric SWR mode peak decreases and the symmetric SWR mode peak significantly increases when one of the $\mathrm{Cu}$ layers is thicker than the other. In this case the asymmetry in the $\mathrm{Cu}$ layers thicknesses ( 3 to 1 ) leads to a strong asymmetry in the $h_{r f}$ field generated in the Py film, and the situation becomes more like the Py film is on the top of (or under) the a single $\mathrm{Cu}$ signal line. The calculated $\mathrm{h}_{\mathrm{rf}}$ field is presented in Fig. 10(a). It is the sum of a nearly uniform field (coming from the currents in the $\mathrm{Cu}$ films) and an antisymmetric field (coming from the currents in Py). As a result, the absorption of the symmetric SWR $(n=1)$ is relatively large. In contrast, if the two $\mathrm{Cu}$ films are equal in thickness, the $\mathrm{h}_{\mathrm{rf}}$ field inside the Py from currents in the $\mathrm{Cu}$ is nearly zero. In this case $\mathrm{h}_{\mathrm{rf}}$ depends only on the small amount of current going through the Py itself, so $h_{\mathrm{rf}}$ is antisymmetric (see Figs. 3, 4, and 6) and the absorption of the symmetric mode is very small.

The situation with the thin $\mathrm{Cu}$ [Fig. 9(b)] is significantly different. Here, the symmetrical SWR mode response is small and does not show substantial changes when the under and over layers have different thicknesses. At the same time, the magnitude of the antisymmetrical SWR peak is large in comparison to the absorption for the symmetric mode. Also, comparing Figs. 9(a) and 9(b) one can clearly see that the measured absorption of the antisymmetrical SWR mode is significantly higher in the case of the thinner $\mathrm{Cu}$ under and over layers.

Note, that in the experiment the FMR peaks linewidth varied from sample to sample. In this case one can use the

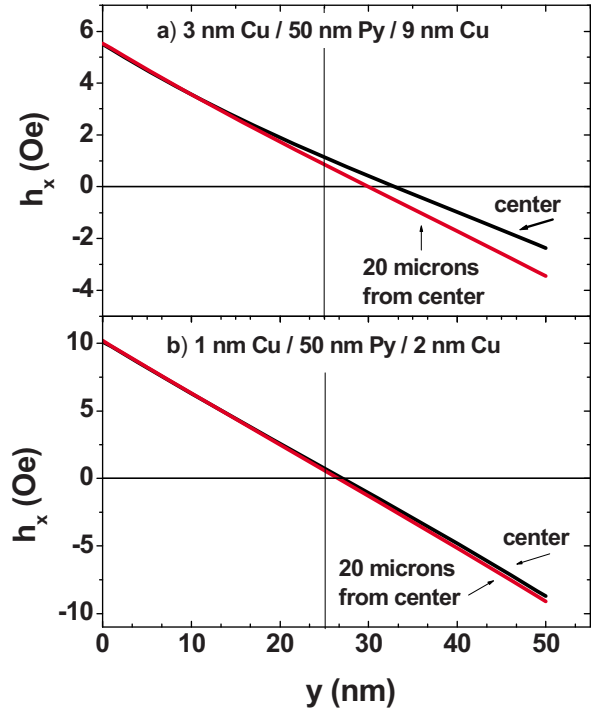

FIG. 10. (Color online) Calculated dependence of $h_{x}$ as function of vertical position $\mathrm{y}$ in the $\mathrm{Py}$ for $\mathrm{x}=0$ (center of signal line) and $\mathrm{x}=20 \mu \mathrm{m}$ (near the edge of the signal line). The frequency is $12 \mathrm{GHz}$ and $\mathrm{H}=0$. (a) $3 \mathrm{~nm} \mathrm{Cu} / 50$ $\mathrm{nm} \mathrm{Py} / 9 \mathrm{~nm} \mathrm{Cu}$ - the thick asymmetric $\mathrm{Cu}$ cladding gives an $\mathrm{h}_{\mathrm{x}}(\mathrm{y})$ signal which is the sum of a constant field and an antisymmetric field. (b) $1 \mathrm{~nm}$ $\mathrm{Cu} / 50 \mathrm{~nm} \mathrm{Py} / 2 \mathrm{~nm} \mathrm{Cu}$ - the thin $\mathrm{Cu}$ cladding gives an $\mathrm{h}_{\mathrm{x}}(\mathrm{y})$ signal which is primarily antisymmetric.

area under the absorption peaks for a more precise comparison of the efficiency of the SWR mode excitation. In Fig. 11, we plot this area as a function of the under and over layers thickness for the samples with $50 \mathrm{~nm}$ thick Py film. One can make three general conclusions as follows:

(1) When the thickness of the Cu layers is small, most of the current will be in the Py layer and $h_{\mathrm{rf}}$ will be antisymmetric about the midplane. As a result, the antisymmetric $(n=2)$ SWR mode is strongly excited and the symmetric SWR mode shows only weak absorption.

(2) When the $\mathrm{Cu}$ layers are thick but equal in thickness the fields from the two $\mathrm{Cu}$ films cancel in the Py. The microwave field $\mathrm{h}_{\mathrm{rf}}$ in the Py comes from the current in the Py and again is antisymmetric. So, again, the antisymmetric $(n=2)$ SWR mode is strongly excited compared

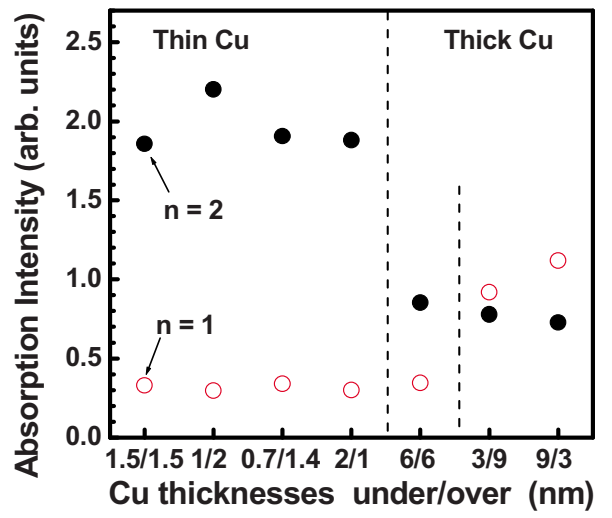

FIG. 11. (Color online) The area under the absorption peaks for the samples with a $50 \mathrm{~nm}$ thick Py layer and different thicknesses of $\mathrm{Cu}$ under and over layers. The open circles correspond to the lowest symmetrical SWR mode response and the filled circles correspond to the lowest antisymmetrical SWR mode response. Data corresponds to measurements performed at a pumping frequency of $12 \mathrm{GHz}$. 
to the symmetric $(n=1)$ mode. However, the absorption for the $n=2$ mode is smaller than for that in the thin $\mathrm{Cu}$ cases due to the fact $h_{\mathrm{rf}}$ is smaller compared to the previous case.

(3) When the $\mathrm{Cu}$ layers are thick and unequal in thickness most of the current is in the thicker $\mathrm{Cu}$ film. The generated microwave field $h_{r f}$ is not purely antisymmetric in the Py film and the symmetric mode is more strongly excited.

Thus the absorption of the SWR modes can be changed by varying the bottom and top layers thicknesses. Nevertheless we were not able to completely suppress the symmetrical SWR mode response by adjusting the thicknesses of the bottom and top layers. One of the possible reasons is that the remaining part of the symmetrical SWR mode response was produced at the edges of the Py signal line where the simple model described above becomes inapplicable.

We note that the observation of a strong $n=2$ mode in Py waveguide structures was a part of the results found for FMR in micrometer-sized Py elements and was originally reported at a conference. ${ }^{26}$ In this work we have performed measurements on a variety of geometries and provide a full analysis of the FMR absorption and the effects of nonmagnetic metallic cladding layers. There have also been some recent measurements indicating similar behavior in microwave stripline waveguides rather than coplanar waveguides ${ }^{27}$ but the majority of that work concentrated on magnetic bilayers structures.

\section{CONCLUSION}

In this paper we have described a new and simple geometry that creates a microwave driving magnetic field which is antisymmetric about the midplane of a ferromagnetic film and is strongly coupled with the antisymmetrical SWR mode for films with symmetrical magnetic pinning conditions. The proposed geometry utilizes a coplanar waveguide structure with a ferromagnetic film embedded into the signal line. We present experimental data for signal lines consisting of $\mathrm{Cu} /$ $\mathrm{Py} / \mathrm{Cu}$ structures with different thicknesses for the $\mathrm{Cu}$ layers. The results show the applicability of this geometry for effective excitation of the lowest antisymmetrical SWR mode. Moreover, it was shown that the microwave absorption for the lowest symmetrical and antisymmetrical SWR modes in such structures can be changed by adjusting the thicknesses of the metallic top and bottom cladding layers. In particular, we find that the coupling to the antisymmetric SWR mode is reduced when the either one or both of the $\mathrm{Cu}$ layers are thick enough so that most of the microwave current flows through them. In contrast, when most of the microwave current flows through the Py film, the coupling to the antisymmetric SWR mode is significant.

\section{ACKNOWLEDGMENTS}

This work was supported by the ARO under Grant No. W911NF-04-1-0247 and NSF under Grant No. DMR 0907063. Y.K. would also like to acknowledge support from RFBR (Grant No. 09-07-12148).

${ }^{1}$ T. G. Phillips and H. M. Rosenberg, Rep. Prog. Phys. 29, 285 (1966).

${ }^{2}$ S. E. Barnes, Adv. Phys. 30, 801 (1981).

${ }^{3}$ Z. Frait and D. Fraitova, in Spin Waves and Magnetic Excitations, edited by A. S. Borovik-Romanov and S. K. Sinha (Elsevier, Amsterdam, 1988), Vol. 2.

${ }^{4}$ B. Heinrich and J. F. Cochran, Adv. Phys. 42, 523 (1993).

${ }^{5}$ Z. Celinski, K. B. Urquhart, and B. Heinrich, J. Magn. Magn. Mater. 166, 6 (1997).

${ }^{6}$ M. Farle, Rep. Prog. Phys. 61, 755 (1998).

${ }^{7}$ S. Zhang, S. A. Oliver, N. E. Israeloff, and C. Vittoria, Appl. Phys. Lett. 70, 2756 (1997)

${ }^{8}$ J. Su, C. S. Tsai, and C. C. Lee, J. Appl. Phys. 87, 5968 (2000).

${ }^{9}$ B. J. Kuanr, R. E. Camley, and Z. Celinski, J. Appl. Phys. 95, 6610 (2004).

${ }^{10}$ C. S. Tsai, J. Y. Li, M. J. Chen, C. C. Yu, Y. Liou, D. S. Hung, Y. D. Yao, and S. F. Lee, J. Magn. Magn. Mater. 282, 57 (2004).

${ }^{11}$ B. J. Kuanr, R. E. Camley, and Z. Celinski, J. Magn. Magn. Mater. 286, 276 (2005)

${ }^{12}$ S. S. Kalarickal, P. Krivosik, M. Wu, C. E. Patton, M. L. Schneider, P. Kabos, T. J. Silva, and J. P. Nibarger, J. Appl. Phys. 99, 093909 (2006).

${ }^{13}$ J.-M. L. Beaujour, W. Chen, A. D. Kent, and J. Z. Sun, J. Appl. Phys. 99, 08N503 (2006)

${ }^{14}$ D.-S. Hung, C. S. Tsai, C.-C. Yu, Y. Liou, S.-F. Lee, P.-C. Chiang, C.-S. Ho, and Y.-D. Yao, J. Magn. Magn. Mater. 304, e118 (2006).

${ }^{15}$ C. Bilzer, T. Devolder, P. Crozat, C. Chappert, S. Cardoso, and P. P. Freitas, J. Appl. Phys. 101, 074505 (2007).

${ }^{16}$ B. J. Kuanr, Y. V. Khivintsev, I. Harward, R. E. Camley, Z. J. Celinski, M. Bedenbecker, and H. H. Gatzen, IEEE Trans. Magn. 43, 2648 (2007).

${ }^{17}$ M. Belmeguenai, T. Martin, G. Woltersdorf, M. Maier, and G. Bayreuther, Phys. Rev. B 76, 104414 (2007).

${ }^{18}$ J.-M. L. Beaujour, A. D. Kent, D. W. Abraham, and J. Z. Sun, J. Appl. Phys. 103, 07B519 (2008).

${ }^{19}$ M. Kostylev, J. Appl. Phys. 106, 043903 (2009).

${ }^{20}$ C. Kittel, Phys. Rev. 110, 1295 (1958); See also R. E. Camley and M. Grimsditch, Phys. Rev. B 22, 5420 (1980).

${ }^{21}$ B. Kuanr, I. R. Harward, D. L. Marvin, T. Fal, R. E. Camley, D. L. Mills, and Z. Celinski, IEEE Trans. Magn. 41, 3538 (2005).

${ }^{22}$ R. E. Camley, Z. Celinski, T. Fal, A. V. Glushchenko, A. J. Hutchison, Y. Khivintsev, B. Kuanr, I. R. Harward, V. Veerakumar, and V. V. Zagorodnii, J. Magn. Magn. Mater. 321, 2048 (2009).

${ }^{23}$ P. E. Wigen, C. F. Kooi, M. R. Shanabarger, and T. D. Rossing, Phys. Rev. Lett. 9, 206 (1962).

${ }^{24}$ M. Nisenoff and R. W. Terhune, J. Appl. Phys. 36, 732 (1965).

${ }^{25}$ S. Y. An, P. Krivosik, M. A. Kraemer, H. M. Olson, A. V. Nazarov, and C. E. Patton, J. Appl. Phys. 96, 1572 (2004).

${ }^{26}$ Y. V. Khivintsev, R. Adam, C. M. Schneider, B. V. McGrath, R. E. Camley, and Z. J. Celinski, in 53rd Magnetism and Magnetic Materials Conference, Abstract CV-02, Austin, Texas, 2008.

${ }^{27}$ M. Kostylev, K. J. Kennewell, R. Magaraggia, R. L. Stamps, M. Ali, and B. J. Hickey, arXiv:0908.4443v2 [cond-mat.mtrl-sci]; R. Magaraggia, M. Kostylev, R. L. Stamps, K. Kennewell, M. Ali, and B. J. Hickey, International Conference on Magnetism, Abstract TH-FW4-03, Karlsruhe, Germany, 2009 (unpublished). 